\title{
Multifocal gastric adenocarcinoma in a patient with LRBA deficiency
}

\author{
Nina Bratanič ${ }^{1}$, Jernej Kovač², Katka Pohar ${ }^{3}$, Katarina Trebušak Podkrajšek ${ }^{2,4}$, Alojz Ihan ${ }^{3}$, Tadej Battelino ${ }^{1,4}$ \\ and Magdalena Avbelj Stefanija ${ }^{{ }^{*}}$ (D)
}

\begin{abstract}
Background: Lipopolysaccharide-responsive, beige-like anchor protein (LRBA) deficiency is characterized by primary immunodeficiency and autoimmunity. Cancer may present another feature of LRBA deficiency. We describe a case history of a young adult with LRBA deficiency and two independent malignancies.

Methods: Family-trio whole exome sequencing with unbiased phenotype ontology approach was used for identification of causative mutations of a primary immune deficiency disorder. Additionally, we sought to identify germline mutations in genes known to be associated with two independent malignancies using a targeted approach. A cytotoxic T-lymphocyte associated protein 4 (CTLA4) expression in T lymphocytes was determined by flow cytometry.
\end{abstract}

Results: In the patient with clinical signs of LRBA deficiency multifocal gastric carcinoma and malignant melanoma were diagnosed and surgically treated at 19 and 27 years of age, respectively. Despite refusal of any adjuvant chemotherapy or radiotherapy, the patient demonstrated disease free survival for at least 13 years after the first cancer diagnosis. A homozygous frameshift deletion in LRBA gene (p.Glu946Ter) and two common variants in TYR gene were identified. Reduced CTLA4 expression in a subset of regulatory T lymphocytes was identified in the patient and his unaffected mother carrying a heterozygous LRBA mutation as compared to control in a dose-dependent manner.

Conclusion: This is the first description of gastric cancer and malignant melanoma in a young adult with LRBA deficiency. The role of $\angle R B A$ gene knockout in cancer development and its prognosis remains to be elucidated.

Keywords: Lipopolysaccharide-responsive, Beige-like anchor (LRBA), Immunodeficiency, Autoimmunity, Gastric cancer, Malignant melanoma, Cytotoxic T-lymphocyte associated protein 4 (CTLA4), Malignancy

\section{Background}

Deleterious germline mutations in $L R B A$ gene encoding lipopolysaccharide-responsive, beige-like anchor protein (LRBA) have been recently associated with an autosomal recessive monogenic disorder, whose common denominators are LRBA deficiency, Autoimmunity, regulatory $\mathbf{T}$ (Treg) cell defects, Autoimmune Infiltration, and Enteropathy (LATAIE syndrome) [1-5]. To our knowledge, more than 60 patients with LRBA deficiency have been reported, with a plethora of diverse mutations identified and with

\footnotetext{
* Correspondence: Magdalena.avbelj@mf.uni-lj.si

'Department of Pediatric Endocrinology, Diabetes and Metabolism,

University Medical Centre, University Children's Hospital, Bohoriceva 20, 1000

Ljubljana, Slovenia
}

Full list of author information is available at the end of the article highly variable clinical and immunologic characteristics [1-18]. Several neoplasms are reported in LRBA deficiency: Burkitt lymphoma [10], low-grade Ebstein Barr virus positive (EBV+) marginal zone lymphoma [17], lymphomatous central nervous system pseudotumor [2], dysplastic tubular adenoma and polyps [18], and immunoproliferative diseases $[4,7,8]$, suggesting that proliferative diseases may present another feature of LRBA deficiency.

We describe clinical, immunologic, and genetic characteristics of a patient with a novel pathogenic homozygous LRBA gene mutation presenting not only with immune deficiency and multiorgan autoimmunity, but also with two independent malignant diseases. The presented clinical spectrum recapitulates and extends the previously described phenotypes. 


\section{Methods}

\section{Patient}

The clinical history of a 32-year old Caucasian male patient, followed up since infancy, is described. Clinical data were obtained from the medical records upon prior patient's written consent. Genetic analysis of his pedigree was performed after the patient and his parents gave written informed consent approved by the Republic of Slovenia National Medical Ethics Committee.

\section{Genetic analysis}

Whole exome sequencing (WES) was performed in Eurofins Genomics (Ebersberg, Germany) using Ion AmpliSeq Exome kit for whole exome enrichment preparation and Ion $\mathrm{PI}^{\mathrm{Ix}}$ Sequencing 200 Kit v3 together with Ion Proton Sequencer (Thermo Fisher Scientific, Waltham, MA, USA).

Genetic variants with coverage $>15 \times$ were analyzed with Variant Studio 2.2 software (Illumina, San Diego, CA, USA). A combination of family trio approach and phenotype driven analysis with Human Phenotype Ontology database [19] was used to direct and focus the analysis on $L R B A$ genetic variants as shown in Additional file 1: Figure S1. The minor allele frequency threshold for known variants was set at $1 \%$, and all variants exceeding this value were excluded from further analysis. Family segregation analysis with De novo and Autosomal Recessive inheritance model was used to further reduce the number of potential causative variants. Identified candidate variant and its family segregation was confirmed by a targeted Sanger sequencing run on ABI Genetic Analyzer 3500 (Applied Biosystems, Waltham, MA, USA) using custom oligonucleotides and BigDye Terminator v3.1 sequencing kit (Applied Biosystems, Waltham, MA, USA). In addition, genes associated with inherited susceptibility to malignant melanoma (CDKN2A, MDM2, CDK4, RB1, MC1R, TYR, TYRP1 and $A S I P)$ [20], gastric cancer (CDH1, CTNNA1, BRCA1, BRCA2, APC, TP53, STK11, SMAD4, BMPR1A, MSH2, $M L H 1$ ) [21], and CTLA4 gene were analyzed with targeted approach.

The Loss of Heterozygosity (LoH) was analyzed to evaluate the potential degree of consanguinity. Using HomSI algorithm [22], the WES data were analyzed for region specific increased ratio of homozygous variants.

\section{Cytotoxic T-lymphocyte associated protein 4 (CTLA4) ex- pression by flow cytometry}

Total and mobilized CTLA4 levels were determined as described by Lo et al. [10]. Briefly, peripheral blood mononuclear cells (PBMC) from the patient, carrier and healthy donor were isolated from whole blood using Ficoll-Paque gradient separation (GE Healthcare, Sweden). The collected cells were resuspended at $1 \times 106$ cells $/ \mathrm{mL}$ in cell culture media (RPMI, 5\% FCS, 1\% penicillin/streptomycin, 1\% L-glutamine). Total CTLA4 levels were determined by fixing and permeabilizing (BioLegend FoxP3 staining kit) freshly isolated cells, staining for CTLA4 and FoxP3 and surface markers, including cluster of differentiation (CD) 3, CD4 and CD25 and analyzed by flow cytometry. Mobilized CTLA4 levels were determined by stimulating the cells in the presence of anti-CTLA4 antibody (BD Biosciences, San Jose, California, USA) with $20 \mathrm{ng} / \mathrm{mL}$ phorbol 12-myristate 13-acetate (PMA) (Sigma-Aldrich, St. Louis, Missouri, USA) and $1 \mu \mathrm{M}$ ionomycin (Sigma-Aldrich, St. Louis, Missouri, USA) for $30 \mathrm{~min}$ at $37{ }^{\circ} \mathrm{C}$ and $5 \%$ carbon dioxide $\left(\mathrm{CO}_{2}\right)$ in the medium. Cells were then washed, fixed, permeabilized and stained as described above.

\section{Results}

\section{Case report}

Propositus was born as the only child in the family in which no cancer or immune deficiency in the family history was reported. Parents are distant relatives; the patient's great grandmother and great grandfather were first cousins. In support to their distant relation larger stretches of LoH regions were identified at chromosomes 2, 5 and 6 (Additional file 2: Figure S2). The patient presented at the age of 3 months with severe protracted diarrhea, hypoproteinemia and anemia. At the age of 18 months, total villous atrophy was found by endoscopic biopsy, but the result of serologic test for celiac disease was negative. A gluten free diet was introduced without significant clinical improvement. In addition to autoimmune enteropathy, the patient developed anti-nuclear antibodies (ANA) positive oligoarticular rheumatoid arthritis at the age of 3 years. Corticosteroid treatment was started which resulted also in a clinical improvement of intestinal manifestations. At the age of 10 years, autoimmune enteropathy was confirmed by gastrointestinal histology of jejunum, where mild villous atrophy and moderate crypt hyperplasia were seen. By immunohistochemistry equal number of plasma cells, immunoglobulin (Ig) A and IgM positive cells and the presence of IgE positive cells in lamina propria were determined; furthermore granular immune complexes along epithelial basal membrane were identified. Another immunohistochemic evaluation of colon biopsy performed at the age of 23 years demonstrated infiltration of mucosa with CD8+ cells and loss of enteroendocrine cells, but eosinophilic infiltrates or CD20+ B cells were not present. Since 3 years of age any attempt to taper steroids led to recurrent chronic diarrhea, so the patient was continuously treated with therapeutic doses of steroids, mostly without a protection with proton pump inhibitors until a diagnose of gastric cancer. Furthermore, malabsorption progressed and short bowel syndrome developed. At the age of 28 years, when his enteral losses increased to $3000 \mathrm{ml}$ per 
day and he started to lose his weight, the patient could be persuaded to start treatment with parenteral nutrition in addition to enteral feeding. Various attempts of systemic or topical immunosuppression with azathioprine and budesonide to treat his enteropathy were of limited and/ or short-term success. After the introduction of treatment with tacrolimus, the quantity of enteral losses diminished from $3000-4000 \mathrm{ml}(125-160 \mathrm{ml} / \mathrm{kg}$ body weight/day) to $1500 \mathrm{ml}$ per day $(62,5 \mathrm{ml} / \mathrm{kg}$ body weight/day), which, after the introduction of abatacept, further diminished to $600 \mathrm{ml}$ per day $(25 \mathrm{ml} / \mathrm{kg}$ body weight/day). Schematic representation of selected therapy and major disease manifestations is shown in Fig. 1.

In addition to autoimmune enteropathy and rheumatoid arthritis, the patient had megaloblastic anemia with vitamin B12 deficiency responsive to monthly intramuscular vitamin B12 injections, primary hypothyroidism and atopic dermatitis. The results of autoantibodies evaluated to diagnose autoimmunity are briefly summarized in Additional file 3: Table S1, but anti-intrinsic autoantibodies were never determined. The patient had extreme growth retardation with final height of $123 \mathrm{~cm}$ with normal growth hormone secretion and was unresponsive to growth hormone therapy. He also had failure to thrive, Cushingoid face, osteoporosis, very thin skin with subcutaneous calcinations, nephrocalcinosis, cholelithiasis, arterial hypertension and a cataract, likely as complications of a long term corticosteroid treatment (and/or chronic disease). The patient was treated with high doses of growth hormone for 7 years and, despite increasing the dose of growth hormone, insulin-like growth factor 1
(IGF-1) and insulin-like growth factor binding globuline 3 (IGFBP3) levels reached only the average age and sex adjusted levels.

During the follow-up, we observed gradual decline in the number of $\mathrm{B}$ cells and immunoglobulin levels. Low $\mathrm{B}$ and $\mathrm{T}$ lymphocytes subpopulations and hypogammaglobulinaemia confirmed immunodeficiency, with low serum IgG and IgM levels and normal IgA. After 26 years of age monthly intravenous replacement of immunoglobulins was required. As shown by flow cytometry almost complete absence of $\mathrm{B}$ cells, markedly reduced number of $\mathrm{T}$ cells, CD4+ and $\mathrm{CD} 8+\mathrm{T}$ cell subsets and Natural killer (NK) cells were demonstrated before the patient's first cancer diagnoses (Additional file 4: Table S2). The number of naive Th (CD45 RA) and $\mathrm{CD} 25+\mathrm{CD} 4+\mathrm{T}$ cells was significantly reduced. $\mathrm{T}$ cell proliferative responses to phytohemagglutinin (PHA) were reduced, but after stimulation with $\mathrm{CD} 3 / \mathrm{CD} 28$ normal $\mathrm{T}$ cell proliferative response was observed. Extensive immunophenotyping in recent years demonstrated that 96\% of T lymphocytes HLA-DR were activated, suggestive of a hyperactive immune state.

The patient suffered from several opportunistic infections, including Campylobacter jejuni, Morganella morganii, Proteus mirabilis, Yersinia enterocolitica, Cryptospordium parvus, Giardia lamblia, Legionella pneumophila, pulmonary aspergillosis and aspergilloma, and chronic mucocutaneous candidiasis. He had immunologically confirmed Ebstein Barr virus primo infection at the age of 21 years. In addition, he had recurrent episodes of catheter sepsis caused by Staphyloccocus epidermidis.

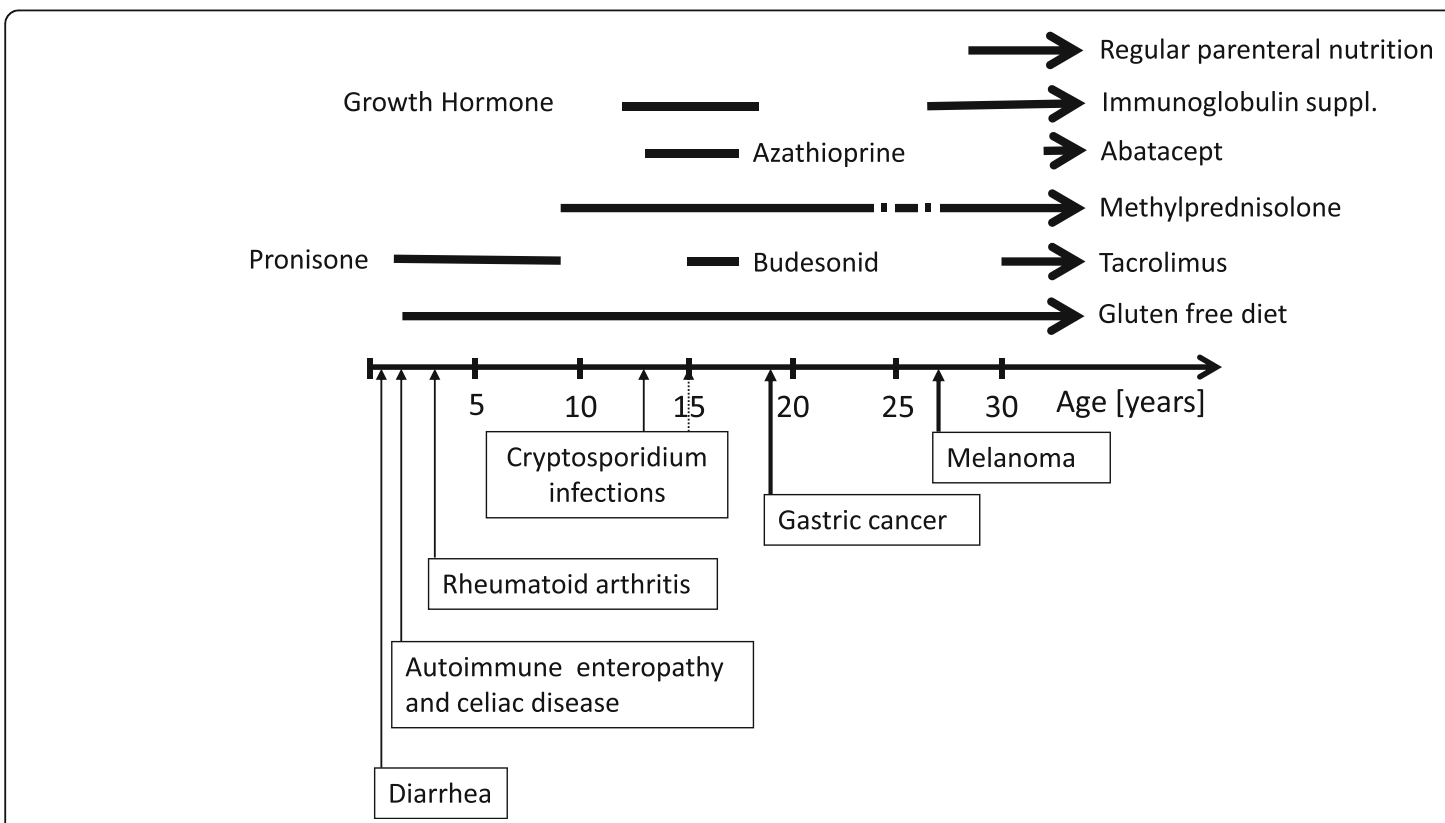

Fig. 1 Schematic representation of the course of selected treatments and age at occurrence of selected disease manifestations. Arrows indicate ongoing therapy. Broken lines indicate two periods of pulse therapy with methyprednisolone 
At the age of 19 years, an abdominal ultrasound revealed a gastric tumor arising from the anterior and posterior wall of the gastric corpus that almost obstructed the outflow (Additional file 5: Figure S3). Subtotal gastrectomy with Roux-en-Y gastrojejunostomy with extended lymph node dissection was performed (1/36 positive). Histopathological findings (Additional file 5: Figure S3) confirmed multifocal invasive gastric adenocarcinoma, arising in a background of two separate adenomas with a high grade dysplasia. Carcinoma was mostly of an intestinal type (Lauren) and of an infiltrative type (Ming). Muscularis propria infiltration (pT2a) and lymphangiosis carcinomatosa were observed. At least two additional focuses of intramucosal carcinoma were identified in the surrounding mucosa. The dysplasia was present in a background of a chronic, diffuse active gastritis with intestinal metaplasia. Immunohistochemical studies for Helicobacter pylori were negative. Further treatment with chemo- and radiotherapy was suggested, which the patient declined. By the time of a publication 13 years later during regular oncological and radiological follow-up, no signs of progression of gastric carcinoma were observed. At the age of 27 years, the patient had excision of a $4 \mathrm{~mm}$ wide malignant melanoma in situ, located at the right ankle. The patient had fair skin and fair hair. No sunburns were reported in his history as well as very little sunlight exposure.

\section{Genetic analysis}

Using whole exome sequencing with phenotype ontology approach a homozygous small frameshift deletion in LRBA (NM_006726.4: c.2836_2839delGAAA; NP006717.2: p.Glu946Ter, rs533294277, minor allele frequency in ExAC database [23] $1,66 \times 10^{-5}$ ) was identified in the patient, confirmed by Sanger sequencing. The mutation introduces a premature stop-codon and is therefore considered pathological. Both parents were heterozygous carriers. The average coverage in region of interest captured by WES was $58.4 \mathrm{X}, 57.2 \mathrm{X}, 53.9 \mathrm{X}$ for the patient, his mother and father, respectively.

Additionally, compound heterozygosity for p.Ser192Tyr (NM_000372.4: c.575C > A, rs1042602) and p.Arg402Gln (c.1205G > A, rs1126809) polymorphisms in the tyrosinase gene $(T Y R)$ (minor allele frequency 0,12 and 0,08 , respectively, according to dbSNP database, [24]) was determined, p.Ser192Tyr being inherited from the father and p.Arg402Gln from the mother. No other potentially pathogenic variants were identified in other melanoma or gastric cancer predisposing genes or in CTLA4 gene.

\section{T cell subtype specific and LRBA dose-dependent reduction of CTLA4}

FoxP3+ cells were assessed on freshly isolated (unstimulated) and stimulated PBMC (stimulation with ionomycin and PMA) by flow cytometry, as described [10]. A markedly reduced CTLA4 expression on Treg cells (CD3+ CD4+ FoxP3+) was demonstrated in the patient, compared to healthy donor (Fig. 2). Furthermore, a reduction of CTLA4 expression in Treg cells was identified also in a heterozygous clinically unaffected mother. The CTLA4 mean fluorescence intensity (MFI) on FOXP3 + CD4+ T cells was highest in the healthy donor, lower in the heterozygous carrier and lowest in the LRBA deficient patient (Table 1). Interestingly, a reduction of CTLA4 expression in the patient and carrier was identified only in FoxP3+ T cells (Treg), while FoxP3 negative CD4 $\mathrm{T}$ cells (non - regulatory $\mathrm{T}$ helper cells) and $\mathrm{CD} 4$ negative $\mathrm{T}$ cells ( $\mathrm{T}$ cytotoxic cells) expressed lower level of CTLA4 expression, and also no difference in the patient, carrier and healthy donor was measured (Table 1).

At the time of CTLA4 expression analysis, the patient was more than six months off abatacept therapy.

\section{Discussion}

Biallelic mutations in $L R B A$ were previously implicated as a cause of common variable immunodeficiency with autoimmunity in humans. Like in many other primary immunodeficiency disorders, there is no clear genotypephenotype correlation in patients with $L R B A$ mutations. Multifocal gastric cancer and malignant melanoma were so far not reported in patients with $L R B A$ gene mutations.

Our patient had several risk factors for developing gastric carcinoma. First, he had a "common variable immunodeficiency (CVID)-like condition" with multiple chronic infections and inflammation. The emergence of malignancies in primary immunodeficiency disorders results from the interplay between the underlying genetic defect, immune dysregulation with defective immunosurveillance mechanisms, and increased susceptibility to specific viruses [25]. Specifically, patients with CVIDs have a 10-fold increased risk of gastric cancer. The mechanisms are not fully understood, but the increased risk has been linked to a number of CVID-associated factors, including pernicious anemia, gastric atrophy, achlorhydria, decreased gastric IgA, and chronic Helicobacter pylori infection [26, 27]. The average age of onset of gastric carcinoma in patients with CVID is earlier than in those without immunodeficiency (46 years vs. 69 years) [28], but still much later then in our patient. Second, prolonged therapy with corticosteroids likely worsened existing immune dysfunction and increased the risk for cancer. Third, our patient had a recurrent infection with Cryptosporidium. The study on dexamethasone treated severe combined immunodeficiency (SCID) mice demonstrated that parasite C. parvum induced digestive adenocarcinoma [29].

The association of elevated growth hormone levels with gastric cancer has not been observed in either 


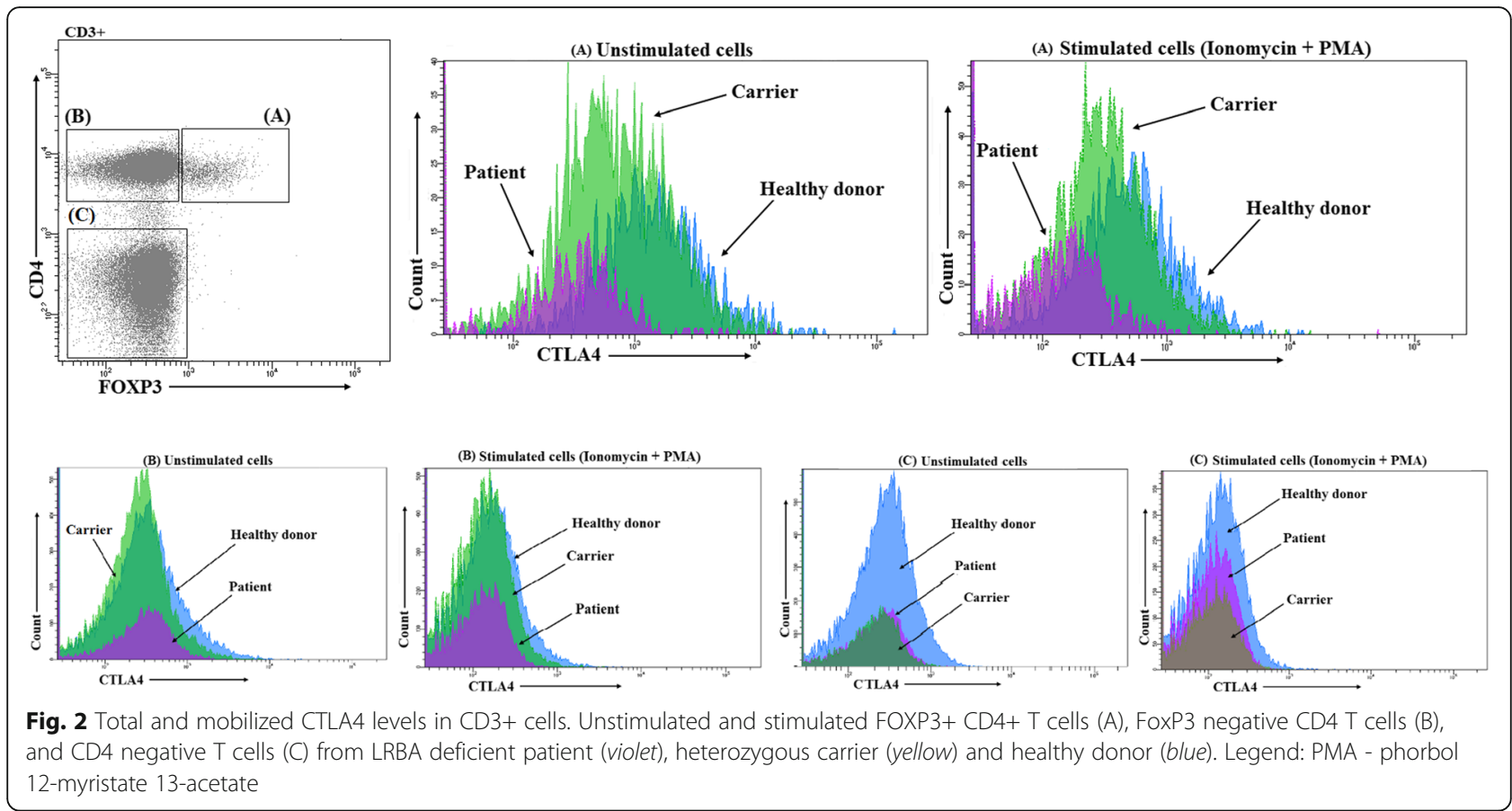

growth hormone treated patients or in patients exposed to extreme endogenous growth hormone secretion due to acromegaly [30].

Multiple cancers at a relatively young age might be associated with inherited cancer predisposition. Particularly, gastric cancer at such an early age is rare and likely attributed to increased sensitivity. Malignant melanoma on the other hand is a common cancer in Slovenian population with a crude incidence rate of $24,1 / 100.000$ in a 5-year interval from 2009 to 2013. Furthermore, it is the most common cancer in Slovenian population younger than 30 years with $4,2 \%$ of all newly diagnosed melanoma patients being younger than 30 years [31]. The patient is a carrier of two common variants in $T Y R$ gene, p.Ser192Tyr and p.Arg402Gln of which only the variant p.Arg402Gln was associated with increased risk for melanoma in one large population study with odds ratio (OR) 1,21 [32], but not in two other similar studies [33, 34]. On the other hand, many primary immunodeficiency disorders are associated with elevated risks for different types of cancer [26]. Therefore, association of malignant diseases in our patient with LRBA deficiency can be suspected.

LRBA plays a major immunoregulatory role by helping maintain intracellular stores of CTLA4, which allows protein to mobilize rapidly to the cell surface [10]. CTLA4 is a critical and potent inhibitor of T-cell proliferation that serves as a "checkpoint" of immune responses. CTLA4 gene haploinsufficiency with autoimmune infiltration (CHAI) leads to broad clinical manifestations such as hypogammaglobulinemia, enteropathy, recurrent infections, lymphocytic infiltration, and multiple autoimmune clinical features, very similar to characteristics of LRBA deficiency [5, 35-37]. Interestingly, 3 of the 24 (12.5\%) cases with CTLA4 haploinsufficiency reported developed gastric cancer and 2 of 3 patients presented with multifocal adenocarcinomas associated with atrophic gastritis and intestinal metaplasia, similarly to our patient [35-37]. We did not identify any potentially pathogenic variants in

Table 1 The quantification of total and mobilized CTLA4

\begin{tabular}{lllll}
\hline Status & & $\begin{array}{l}\text { Mean Fluorescence Intensity (MFI) } \\
\text { of CTLA4 on FOXP3+ CD4+ T cells }\end{array}$ & $\begin{array}{l}\text { MFI of CTLA4 on } \\
\text { FOXP3- CD4+ T cells }\end{array}$ & $\begin{array}{l}\text { MFI of CTLA4 on } \\
\text { CD3 + CD8+ T cells }\end{array}$ \\
\hline HEALTHY DONOR & UNSTIMULATED & 2340 & 490 & 315 \\
& IONO + PMA & 695 & 178 & 119 \\
CARRIER & UNSTIMULATED & 1152 & 324 & 214 \\
& IONO + PMA & 393 & 121 & 89 \\
PATIENT & UNSTIMULATED & 501 & 331 & 215 \\
& IONO + PMA & 176 & 106 & 85 \\
\hline
\end{tabular}


propositus's CTLA4 gene, but a markedly reduced CTLA4 expression by Treg cells was demonstrated by flow cytometry, as demonstrated previously in LRBA deficient patients [10]. Furthermore, a reduction of CTLA4 expression in Treg cells was identified also in a heterozygous clinically unaffected mother, which is a demonstration of an unopposed gene dosage effect of LRBA gene mutations in vivo. Even so Treg CTLA4 expression reduced by half seems to be clinically silent. Interestingly, CTLA4 gene haploinsufficiency is disease-causing, while $L R B A$ gene haploinsufficiency state in propositus's mother caused no apparent disease until her current age of 54 years. Of note, no reduction in CTLA4 expression in the patient as compared to the control was demonstrated in FoxP3 negative CD4 $\mathrm{T}$ cells (non - regulatory $\mathrm{T}$ helper cells) and CD4 negative $\mathrm{T}$ cells (cytotoxic $\mathrm{T}$ cells). This suggests LRBA has a T cell subtype specific influence on CTLA4 expression, which may have importance in the disease mechanism. Both cell populations expressed lower level of CTLA4 expression. Differential CTLA4 expression in human CD4+ versus CD8+ T cells was described recently demonstrating significantly higher CTLA4 expression in the CD4+ T cells than in CD8+ T cells [38]. CTLA4 is higher at the protein and the transcriptional level in CD4+ T cells. These findings demonstrate a differential regulation of CTLA4 on CD4+ and CD8+ T cell subsets, which is likely important to the clinical efficacy for anti-CTLA4 therapies.

Intriguingly, LRBA deficiency and CTLA4 haploinsufficiency could represent human in vivo models of CTLA4 blockade with checkpoint inhibitors; medicines that actually improved survival in several cancers, particularly in melanoma, but also in gastric cancer $[39,40]$. CTLA4 serves as a critical checkpoint inhibitor as it downregulates $\mathrm{T}$ cell activation to prevent autoimmunity and allow tolerance to self-antigens. Wang JW et al. showed that LRBA is significantly upregulated in multiple tumor types [41]. Its expression and function are important for cancer cell proliferation and apoptosis. Inhibition of LRBA function may repress cancer cell growth in many cancer cell lines, but not all. These findings suggest that LRBA knockdown, when combined with cancer therapeutics, may achieve greater therapeutic effect than either entity alone. According to Wang JW et al. [41] we would expect that these patients are protected against cancer. Of note, our patient declined chemotherapy, yet he survived more than 13 years without reappearance of the disease, which is significantly longer survival than expected according to reports. Little is known concerning the long-term survival of adolescents and young adults with solid gastric cancers. Survival of 21 patients (12 females, 9 males) between 5 and 21 years of age ranged from 1 to 25 months [42]. In general, survival of immunodeficiency-associated gastric cancer in adult populations is longer, but still rarely over 10 years [28].

\section{Conclusions}

In conclusion, to the best of our knowledge, we present the first co-occurrence of gastric cancer and malignant melanoma with LRBA deficiency. Despite the risk factors, including multifocal gastric cancer with muscularis propria invasion, lymphangiosis carcinomatosa, a positive lymph node, young age of onset, additional cancer and immunodeficiency, our patient demonstrated long cancerfree survival with only surgical treatment. The possible role of $L R B A$ gene knockout in cancer development and its prognosis remains to be elucidated. The identification of key molecules that regulate cellular immune processes may enable development of novel immunotherapeutic approaches to cancer treatment.

\section{Additional files}

Additional file 1: Figure S1. Workflow of WES filtering process with multiple filtering steps including a phenotype driven filtering. (PDF 308 kb)

Additional file 2: Figure S2. The identified regions of homozygous stretches in chromosome 2,5 and 6. Description: Blue regions indicate homozygous variants and yellow regions indicate heterozygous variants. Orange regions in parental chromosomes (F, M) indicate heterozygous variants corresponding with homozygous variants of sibling's genotype (P). (TIFF 898 kb)

Additional file 3: Table S1. Autoantibodies evaluated in the patient. (DOCX $12 \mathrm{~kb}$ )

Additional file 4: Table S2. Selected immune system determinants longitudinally evaluated in the patient. (DOCX $15 \mathrm{~kb}$ )

Additional file 5: Figure S3. Ultrasound and histopathologic images of the gastric cancer. Description: (a.) Ultrasound image of a $49 \times 44 \mathrm{~mm}$ large tumor formation in the stomach; (b., c.) histopathologic images of bioptic material from the gastric tumor demonstrating intestinal type gastric carcinoma with lamina propria invasion (Kreyberg trichrom stain, 20X magnification (b.) 100X magnification (c.)). The square in part b. indicates the area of magnification in part c. of the figure. (PDF $223 \mathrm{~kb}$ )

\section{Abbreviations}

ANA: Anti-nuclear autoantibodies; CD: Cluster of differentiation; CHAl: CTLA4 gene Haploinsufficiency with Autoimmune Infiltration; CTLA4: Cytotoxic T-lymphocyte associated protein 4; CO2: Carbon dioxide; CTLA4: Cytotoxic T-lymphocyte associated protein 4; CVID: Common variable immunodeficiency; DNA: Deoxyribonucleic acid; EBV: Ebstein Barr virus; EDTA: Ethylenediaminetetraacetic acid; Ig: Immunoglobulin; IGF-1: Insulin-like growth factor 1; IGFBP3: Insulin-like growth factor binding globulin 3; LATAIE: Autoimmunity, regulatory T (Treg) cell defects, Autoimmune Infiltration, and Enteropathy; LoH: Loss of Heterozygosity; LRBA: Lipopolysaccharideresponsive, beige-like anchor protein; MFI: Mean fluorescence intensity; NK: Natural killer cell; OR: Odds ratio; PBMC: Peripheral blood mononuclear cells; PHA: Phytohemagglutinin; PMA: Phorbol 12-myristate 13-acetate; SCID: Severe combined immunodeficiency; WES: Whole exome sequencing

\section{Acknowledgments}

We thank the patient and his family that kindly agreed with the publication of their data. We also thank prof. dr. Jože Pižem for his expert opinion on histopathologic results.

\section{Funding}

This work was supported by the Slovenian Research Agency, grant No. P3-0343.

\section{Availability of data and materials}

The data supporting the conclusions of this article are included within the article and its additional files. Additional datasets used and/or analyzed during the current study are available from the corresponding author on reasonable request. 


\section{Authors' contributions}

NB analyzed and interpreted the patients data regarding the immune deficiency and cancer, and was a major contributor in writing the manuscript. JK and KTP analyzed and interpreted the genetic information of the pedigree. KP and Al performed and interpreted the CTLA4 expression analysis and reviewed the manuscript. TB and MAS participated in writing the manuscript. All authors read and approved the final manuscript.

\section{Ethics approval and consent to participate}

The patient and his parents gave written consent for molecular-genetic analysis on local consent forms approved by the Medical Ethics Committee of the Republic of Slovenia (\#28/04/10 and \#132/03/15).

\section{Consent for publication}

The patient gave a written consent for publication on a local form approved by the Medical Ethics Committee of the Republic of Slovenia (\#132/03/15).

\section{Competing interests}

The authors declare that they have no competing interests.

\section{Publisher's Note}

Springer Nature remains neutral with regard to jurisdictional claims in published maps and institutional affiliations.

\section{Author details}

'Department of Pediatric Endocrinology, Diabetes and Metabolism, University Medical Centre, University Children's Hospital, Bohoriceva 20, 1000 Ljubljana, Slovenia. 'University Medical Centre, University Children's Hospital, Unit for Special Laboratory Diagnostics, Vrazov trg 1, 1000 Ljubljana, Slovenia. ${ }^{3}$ University of Ljubljana, Faculty of Medicine, Institute for Microbiology and Immunology, Zaloška 4, 1000 Ljubljana, Slovenia. ${ }^{4}$ University of Ljubljana, Faculty of Medicine, Vrazov trg 2, 1000 Ljubljana, Slovenia.

\section{Received: 30 March 2017 Accepted: 7 July 2017}

\section{Published online: 18 July 2017}

\section{References}

1. Charbonnier LM, Janssen E, Chou J, Ohsumi TK, Keles S, Hsu JT, et al. Regulatory T-cell deficiency and immune dysregulation, polyendocrinopathy, enteropathy, X-linked-like disorder caused by loss-of-function mutations in LRBA. J Allergy Clin Immunol. 2015;135:217-27.

2. Lopez-Herrera G, Tampella G, Pan-Hammarstrom Q, Herholz P, Trujillo-Vargas CM, Phadwal $\mathrm{K}$, et al. Deleterious mutations in LRBA are associated with a syndrome of immune deficiency and autoimmunity. Am J Hum Genet. 2012;90:986-1001.

3. Burns SO, Zenner HL, Plagnol V, Curtis J, Mok K, Eisenhut M, et al. LRBA gene deletion in a patient presenting with autoimmunity without hypogammaglobulinemia. J Allergy Clin Immunol. 2012;130:1428-32.

4. Alangari A, Alsultan A, Adly N, Massaad MJ, Kiani IS, Aljebreen A, et al. LPS-responsive beige-like anchor (LRBA) gene mutation in a family with inflammatory bowel disease and combined immunodeficiency. J Allergy Clin Immunol. 2012;130:481-8.e2

5. Lo B, Fritz JM, SU HC, Uzel G, Jordan MB, Lenardo JM. CHAl and LATIE: new genetic diseases of CTLA-4 checkpoint insufficiency. Blood. 2016;128:1037-42

6. Serwas NK, Kansu A, Santos-Valente E, Kuloglu Z, Demir A, Yaman A, et al. Atypical manifestation of LRBA deficiency with predominant IBD-like phenotype. Inflamm Bowel Dis. 2015;21:40-7.

7. Seidl MG, Hirschmugl T, Gamez-Diaz L, Schwinger W, Serwas N, Deutschmann A et al. Long term remission after allogenic hematopoetic stem cell transplantation in LPS-responsive beige-like anchor (LRBA) deficiency. J Allergy Clin Immunol. 2015;135:1384-90.

8. Revel-Vilk S, Fischer U, Keller B, Nabhani S, Gamez-Diaz L, Rensing-Ehl A, et al. Autoimmune lymphoproliferative syndrome-like disease in patients with LRBA mutation. Clin Immunol. 2015;159:84-92.

9. Alkhairy OK, Abolhassani H, Rezael N, Fang M, Andersen KK, Chavoshzadeh Z, et al. Spectrum of phenotypes associated with mutations in LRBA. J Clin Immunol. 2016:36:33-45.

10. Lo B, Zhang K, Lu W, Zheng L, Zhang Q, Kanellopoulou C, et al. Autoimmune disease. Patients with LRBA deficiency show CTLA4 loss and immune dysregulation responsive to abatacept therapy. Science. 2015;349:436-40.
11. Gámez-Díaz L, August D, Stepensky P, Revel-Vilk S, Seidel MG, Noriko M, et al. The extended phenotype of LPS-responsive beige-like anchor protein (LRBA) deficiency. J Allergy Clin Immunol. 2016;137:223-30.

12. Schreiner F, Plamper M, Dueker G, Schoenberger S, Gámez-Díaz L, Grimbacher B, et al. Infancy-onset T1DM, short stature and severe immunodysregulation in two siblings with a homozygous LRBA-mutation. J Clin Endocrinol Metab. 2016;101:898-904.

13. Lévy E, Stolzenberg M-C, Bruneau J, Breton S, Neven B, Sauvion S, et al. LRBA deficiency with autoimmunity and early onset chronic erosive polyarthritis. Clin Immunol. 2016;168:88-93.

14. Bakhtiar S, Ruemmele F, Charbit-Henrion F, Lévy E, Rieux-Laucat F, Cerf- Bensussan N, et al. Atypical manifestation of LPS-responsive beige-like anchor deficiency syndrome as an autoimmune endocrine disorder without enteropathy and immunodeficiency. Front Pediatr. 2016;4:1-8.

15. Al-Mayouf SM, Naji H, Alismail K, Alazami AM, Sheikh F, Conca W, Al-Mousa H. Evolving spectrum of LRBA deficiency-associated chronic arthritis: is there a causative role in juvenile idiopathic arthritis? Clin Exp Rheumatol. 2017:35:327-9.

16. Shokri S, Nabavi M, Hirschmugl T, Aghamohammadi A, Arshi S, Bemanian MH, et al. LPS-responsive beige-like anchor gene mutation associated with possible Bronchiolitis Obliterans organizing pneumonia associated with Hypogammaglobulinemia and normal lgM phenotype and low number of B cells. Acta Med Iran. 2016;54:620-3.

17. Piquer M, de Valles G, González E, Esteve A, Martín-Mateos MA, Aróstegui الנ, et al. New LRBA-mutation in a patient with severe reduction in $\lg G$, IgM and IgA with normal number of B-cells at diagnosis and previously classified as CVID. J Clin Immunol. 2014;34(Suppl 2):S419.

18. Tessi B, Priftakis $P$, Lindgren F, Chiang SCC, Kartalis N, Löfsted A, et al. Successful Hematopoietic Stem Cell Transplantation in a Patient with LPS-Responsive Beige-Like Anchor (LRBA) Gene Mutation. J Clin Immunol. 2016;36:480-9.

19. Human Phenotype Ontology database. http://www.human-phenotypeontology.org. Accessed 15 June 2016.

20. DermNet New Zealand. Genetics of melanoma. www.dermnetnz.org/ pathology/melanoma-genetics.html. Accessed 4 Apr 2016.

21. Kramer K, Wolf S, Mayer B, Schmidt SA, Agaimy A, Henne-Bruns D, et al. Frequence, Spectrum and prognostic impact of additional malignancies in patients with gastrointestinal Stromal tumors. Neoplasia. 2015;17:134-40.

22. Görmez Z, Bakir-Gungor B, Sağıroğlu MS. HomSl: a homozygous stretch identifier from next-generation sequencing data. Bioinformatics. 2014;30(3):445-7.

23. ExAC Browser (Beta), Exome Aggregation Consortium. http://exac. broadinstitute.org/. Accessed 25 May 2017.

24. dbSNP database. http://www.ncbi.nIm.nih.gov/snp. Accessed 10 February 2017.

25. Rezaei N, Hedayat M, Aghamohammadi A, Nichols KE. Primary immunodeficiency diseases associated with increased susceptibility to viral infections and malignancies. J Allergy Clin Immunol. 2011;127:1329-41.

26. Dhalla F, da Silva SP, Lucas M, Travis S, Chapel H. Review of gastric cancer risk factors in patients with common variable immunodeficiency disorders, resulting in a proposal for a surveillance programme. Clin Exp Immunol. 2011;165:1-7.

27. Agarwal S, Mayer L. Diagnosis and treatment of gastrointestinal disorders in patients with primary immunodeficiency. Clin Gastroenterol Hepatol. 2013:11:1050-63.

28. De Petris G, Dhungel BM, Chen L, Chang Y-HH. Gastric Adenocarcinoma in common variable immunodeficiency: features of cancer and associated gastritis may be characteristic of the condition. Int J Surg Pathol. 2014:22:600-6

29. Benamrouz S, Guyot K, Gazzola S, Mouray A, Chassat T, Delaire B, et al. Cryptosporidium parvum Infection in SCID mice infected with only one Oocyst: GPCR assessment of parasite replication in tissues and development of digestive cancer. PLoS One. 2012;7:e 51232.

30. Clayton PE, Banerjee I, Murray PG, Renehan AG. Growth hormone, the insulin-like growth factor axis, insulin and cancer risk. Nat Rev Endocrinol. 2011;7:11-24.

31. Cancer registry of Republic of Slovenia. http://www.slora.si. Accessed 15 Sept 2016.

32. Gudbjartsson DF, Sulem P, Stacey SN, Goldstein AM, Rafnar T, Sigurgeirsson B, et al. ASIP and TYR pigmentation variants associate with cutaneous melanoma and basal cell carcinoma. Nat Genet. 2008;40:886-91. 
33. Nan H, Kraft P, Hunter DJ, Han J. Genetic variants in pigmentation genes, pigmentary phenotypes, and risk of skin cancer in Caucasians. Int J Cancer. 2009;125:909-17.

34. Ibarrola-Villava M, Hu HH, Guedj M, Fernandez LP, Descamps V, Basset-Seguin N, et al. MC1R, SLC45A2 and TYR genetic variants involved in melanoma susceptibility in southern European populations: results from a meta-analysis. Eur J Cancer. 2012;48:2183-91.

35. Schubert D, Bode C, Kenefeck R, Hou TZ, Wing JB, Kennedy A, et al. Autosomal dominant immune dysregulation syndrome in humans with CTLA4 mutations. Nat Med. 2014;20:1410-6.

36. Hayakawa S, Okada S, Tsumura M, Sakata S, Ueno Y, Imai K, et al. A patient with CTRLA-4 Haploinsufficiency presenting gastric cancer. J Clin Immunol. 2016;36:28-32

37. Zeissig S, Petersen BS, Tomczak M, Melum E, Huc-Claustre E, Dougan SK, et al. Early-onset Crohn's disease and autoimmunity associated with a variant in CTLA-4. Gut. 2014;64:1889-97.

38. Chan DV, Gibson HM, Aufiero BM, Wilson AJ, Hafner MS, Mi QS, Wong HK. Differential CTLA-4 expression in human CD4+ versus CD8+ T cells is associated with increased NFAT1 and inhibition of CD4+ proliferation. Genes Immun. 2014;15:25-32

39. Hodi FS, O'Day SJ, McDermott DF, Weber RW, Sosman JA, Haanen JB, et al. Improved survival with ipilimumab in patients with metastatic melanoma. N Engl J Med. 2010;363:711-23.

40. Kourie HR, Tabchi S, Ghosn M. Checkpoint inhibitors in gastrointestina cancers: expectations and reality. World J Gastroenterol. 2017;23:3017-21.

41. Wang JW, Gamsby JJ, Highfill SL, Mora LB, Bloom GC, Yeatman TJ, et al. Deregulated expression of LRBA facilitates cancer cell growth. Oncogene. 2004:23:4089-97.

42. Sasaki H, Sasano H, Ohi R, Imaizumi M, Shineha R, Nakamura M, et al. Adenocarcinoma at the esophageal gastric junction arising in an 11-year-old girl. Pathol Int. 1999;49:1109-13.

\section{Submit your next manuscript to BioMed Central and we will help you at every step:}

- We accept pre-submission inquiries

- Our selector tool helps you to find the most relevant journal

- We provide round the clock customer support

- Convenient online submission

- Thorough peer review

- Inclusion in PubMed and all major indexing services

- Maximum visibility for your research

Submit your manuscript at www.biomedcentral.com/submit 